\title{
NOTIZEN
}

\section{Beiträge zur Chemie des Phosphors, $71^{1}$ Zur Existenz von Phosphor-selenid-tribromid $\mathrm{PSeBr}_{3}$}

Contributions to the Chemistry of Phosphorus, $71^{1}$ About the Existence of Phosphorus Selenide Tribromide $\mathrm{PSeBr}_{3}$

\section{Marianne Baudler und Bernd Volland \\ Institut für Anorganische Chemie der Universität Köln}

(Z. Naturforsch. 32b, 347-348 [1977]; eingegangen am 26. November 1976)

Phosphorus Selenide Tribromide, Phosphorus Tribromide, Selenium

The title compound is formed in low concentration by addition of selenium to $\mathrm{PBr}_{3}$ or by reaction of $\left(\mathrm{CH}_{3} \mathrm{Se}\right) \mathrm{PBr}_{2}$ with bromine.

Während bei den Phosphor-oxid-halogeniden $\mathrm{POX}_{3}$ und den Phosphor-sulfid-halogeniden $\mathrm{PSX}_{3}$ alle Verbindungen für $\mathrm{X}=\mathrm{F}, \mathrm{Cl}, \mathrm{Br}, \mathrm{I}$ bekannt sind 2, 3, konnte von den Phosphor-selenid-halogeniden $\mathrm{PSeX}_{3}$ bisher nur ein Vertreter, nämlich $\mathrm{PSeI}_{3}{ }^{4}$, dargestellt werden. Im folgenden wird über den Nachweis der Existenz von Phosphor-selenid-tribromid, $\mathrm{PSeBr}_{3} \mathbf{1}$, berichtet.

\section{Reaktion von Phosphor(III)-bromid mit Selen}

Bei der Einwirkung von überschüssigem roten Selen auf Phosphor(III)-bromid in siedendem Schwefelkohlenstoff erfolgt im allgemeinen keine nachweisbare Reaktion sondern lediglich eine Modifikationsänderung des Selens, das praktisch quantitativ zurückgewonnen wird.

Durch systematische Variation der Umsetzungsbedingungen (Dauer der Selenzugabe, Solvensmenge, Reaktionszeit) konnte bei einigen Versuchen ein etwa 25-proz. Umsatz zu 1 erzielt werden (näheres siehe ,Experimentelles“):

$$
\mathrm{PBr}_{3}+\mathrm{Se} \rightleftharpoons \mathrm{PSeBr}_{\mathbf{1}}
$$

Nach Beendigung der Reaktion liegt eine orangefarbene Lösung vor, die nach Abtrennung des nichtumgesetzten Selens $(\approx 75 \%$ der stöchiometrischen Menge) im ${ }^{31} \mathrm{P}-\mathrm{NMR}$-Spektrum neben dem Sin-

Sonderdruckanforderungen an Prof. Dr. M. BAUdLER, Institut für Anorganische Chemie der Universität Köln, Greinstraße 6, D-5000 Köln 41. gulett für $\mathrm{PBr}_{3}$ ein zusätzliches Signal bei - 106,5 $\mathrm{ppm}^{5}$ aufweist. Daß dieses Verbindung 1 entspricht, geht aus dem Vergleich der chemischen Verschiebung mit den betreffenden Werten von $\mathrm{POBr}_{3}\left(-103 \mathrm{ppm}^{6}\right)$ und $\mathrm{PSBr}_{3}\left(-112 \mathrm{ppm}^{6}\right)$ hervor. Beim Entfernen des Lösungsmittels tritt eine weitgehende Zersetzung von 1 unter Abscheidung von elementarem Selen ein. Zahlreiche, unter ähnlichen Bedingungen - auch mit Zusatz von $\mathrm{AlBr}_{3}$ durchgeführte Versuche ergaben nur jeweils einen Umsatz zwischen 1 und $9 \%$.

\section{Reaktion von Methyl-selenyl-phosphordibromid mit Brom}

Nachdem vor kurzem $\mathrm{POI}_{3}$ von KIrSanow und Mitarb. ${ }^{3}$ aus Methoxyphosphordijodid und Jod erhalten werden konnte, war es naheliegend, die Bildung von 1 nach der analogen Reaktion von Methyl-selenyl-phosphordibromid, $\mathrm{CH}_{3} \mathrm{SePBr}_{2}$ 2, und Brom zu untersuchen.

Die bislang unbekannte Verbindung 2 wurde durch Umsetzung von Phosphor(III)-bromid mit Methylselenol erhalten. Das gleichzeitig gebildete Bis(methyl-selenyl)-phosphorbromid, $\left(\mathrm{CH}_{3} \mathrm{Se}\right)_{2} \mathrm{PBr}$, kann durch Thermolyse bei $+30^{\circ} \mathrm{C}$ entfernt werden. 2 ist eine gelbe Flüssigkeit von widerlichem Geruch, die schon in geringen Konzentrationen starke Kopfschmerzen verursacht. Im ${ }^{31} \mathrm{P}-\mathrm{NMR}$ Spektrum zeigt sie ein Signal bei $+210 \mathrm{ppm}^{5} \mathrm{mit}$ den charakteristischen Selen-Satelliten.

Bei der Bromierung von 2 in $n$-Pentan gemäß der Gleichung

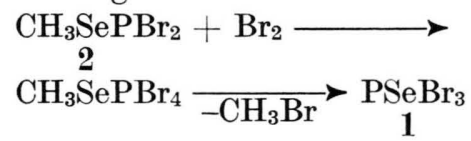

werden bei Raumtemperatur oder $0^{\circ} \mathrm{C}$ neben Methylbromid nur die Zersetzungsprodukte von 1, Phosphor(III)-bromid und elementares Selen, gebildet. Bei $-60^{\circ} \mathrm{C}$ dagegen fällt nur wenig Selen aus, und in der gebildeten dunkelroten Lösung sind ${ }^{31} \mathrm{P}$-NMR-spektroskopisch vier Reaktionsprodukte (in der Reihenfolge abnehmender Konzentration) nachweisbar:

Methyl-selen-phosphonsäure-dibromid,

$\mathrm{CH}_{3} \mathrm{P}(\mathrm{Se}) \mathrm{Br}_{2}\left(\delta=-17,5 \mathrm{ppm}^{7}\right)$;

Phosphor(III)-bromid $\left(\delta=+223 \mathrm{ppm}^{6}\right)$;

Methyl-selenyl-phosphortetrabromid,

$\mathrm{CH}_{3} \mathrm{SePBr}_{4}(\delta=-125 \mathrm{ppm})$;

Phosphor-selenid-tribromid (1) ( $\delta=-108 \mathrm{ppm})$.

Die Zuordnung des Signals bei - $125 \mathrm{ppm}$ zu der Additionsverbindung $\mathrm{CH}_{3} \mathrm{SePBr}_{4}$ ergibt sich aus der Ähnlichkeit der chemischen Verschiebung mit der- 
jenigen von $\mathrm{PBr}_{5}\left(-101 \mathrm{ppm}^{6}\right)$. Demnach wird bei der Reaktion von 2 mit Brom bei $-60^{\circ} \mathrm{C}$ Phosphorselenid-tribromid (1) gebildet, doch ist die Umlagerung von $\mathbf{2}$ in Methyl-selen-phosphonsäure-dibromid demgegenüber stark begünstigt. Versuche zur Auftrennung des Substanzgemisches führten stets zur Zersetzung unter Abscheidung von Selen.

\section{Experimentelles}

Die Arbeiten wurden unter Ausschluß von Licht sowie von Luft und Feuchtigkeit in einer Atmosphäre von gereinigtem Argon ausgeführt. Die verwendeten Lösungsmittel waren getrocknet und Argesättigt. ${ }^{31} \mathrm{P}-\mathrm{NMR}-$ Spektren: Jeol-Kernresonanzspektrometer JNM-C-60 HL, Meßfrequenz $24,3 \mathrm{MHz}$.

\section{Reaktion von $\mathrm{PBr}_{3}$ mit Se}

$2,71 \mathrm{~g}$ (10 mMol) $\mathrm{PBr}_{3}$ und 2,13 $\mathrm{g}$ frisch dargestelltes, fein verteiltes rotes Selen ${ }^{8}$ wurde in $500 \mathrm{ml}$ $\mathrm{CS}_{2}$ zum Sieden erhitzt. Dann wurden unter Kochen am Rückfluß und Rühren eine Lösung von $0,24 \mathrm{~g}$

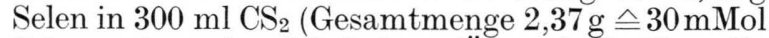
Selen entsprechend 2 -fachem Überschuß über die stöchiometrische Menge) innerhalb von 3 Stdn. zugetropft. Anschließend wurde zur Vervollständigung der Reaktion noch 1 Std. weiter erhitzt. Nach Abziehen des Lösungsmittels i. Vak. bis auf etwa $30 \mathrm{ml}$ wurde das nicht-umgesetzte Selen abgesaugt

1 70. Mitt.: M. Baudler u. B. Carlsohn, Chem. Ber., im Druck.

2 Gmelins Handbuch der Anorganischen Chemie, 8. Aufl., Phosphor, Teil C, Verlag Chemie GmbH, Weinheim 1965.

3 W. G. Kostina, N. G. Feshchenko u. A. W. Kirsanow, Zh. Obshch. Khim. 43, 209 [1973].

${ }^{4}$ M. Baudler, B. Volland u. H.-W. Valpertz, Chem. Ber. 106, 1049 [1973].
$(2,19 \mathrm{~g} \triangleq 27,7 \mathrm{mMol})$ und das Filtrat ${ }^{31} \mathrm{P}-\mathrm{NMR}$ spektroskopisch untersucht. Bezüglich der Ergebnisse siehe S. 347.

\section{Reaktion von Methyl-selenyl-phosphordibromid (2) mit Brom}

Zur Darstellung von 2 wurden 27,1 g (0,1 Mol) $\mathrm{PBr}_{3}$ in $200 \mathrm{ml} n$-Pentan bei $-20{ }^{\circ} \mathrm{C}$ unter Rühren und Durchleiten eines schwachen Argonstromes innerhalb von $8 \mathrm{Stdn}$. mit einer Lösung von $9,5 \mathrm{~g}$ $(0,1 \mathrm{Mol})$ Methylselenol in $100 \mathrm{ml}$ Pentan umgesetzt. Nach Abziehen des Lösungsmittels i. Vak. wurde als Nebenprodukt gebildetes $\left(\mathrm{CH}_{3} \mathrm{Se}\right)_{2} \mathrm{PBr}$ bei $+30{ }^{\circ} \mathrm{C}$ zersetzt und dabei ausgefallenes Selen abgesaugt. Das Filtrat bestand nach ${ }^{31} \mathrm{P}-\mathrm{NMR}$ spektroskopischer Untersuchung aus etwa $95 \mathrm{Mol}-\%$ 2 und 5 Mol- $\% \mathrm{PBr}_{3}$.

$\mathrm{Zu} 14,3 \mathrm{~g}$ des Gemisches aus 2 und $\mathrm{PBr}_{3}$ in $100 \mathrm{ml}$ $n$-Pentan wurde bei $-60{ }^{\circ} \mathrm{C}$ unter intensivem Rühren langsam eine Lösung von $8 \mathrm{~g}$ Brom in $30 \mathrm{ml}$ Pentan getropft. Dann wurde die dunkelrote Reaktionslösung filtriert, auf $80 \mathrm{ml}$ eingeengt und ${ }^{31} \mathrm{P}-\mathrm{NMR}$-spektroskopisch untersucht. Ergebnisse siehe S. 347.

Wir danken dem Minister für Wissenschaft und Forschung des Landes Nordrhein-Westfalen und dem Fonds der Chemischen Industrie für die finanzielle Unterstützung dieser Arbeit.

5 Bezogen auf 85-proz. $\mathrm{H}_{3} \mathrm{PO}_{4}$ (negatives Vorzeichen bei Hochfeldverschiebung).

6 V. Mark, C. H. Dungan, M. M. Crutchfield u. J. R. van Wazer, Topics in Phosphorus Chemistry, Bd. 5, Wiley-Interscience, New York-LondonSidney 1967.

7 L. Maier, Helv. Chim. Acta 46, 2667 [1963].

8 O. Hönigschmid u. W. Kapfenberger, Z. Anorg. Allg. Chem. 212, 198 [1933]. 